\title{
Heating of Large-Area Substrates for In Situ Deposition of YBCO
}

\author{
Alvin J. Drehman, John S. Derov, Jane A. Horrigan, Robert J. Andrews, and Derek S. Linden \\ Rome Laboratory RL/ER, 80 Scott Drive , Hanscom AFB, MA 01731-2909
}

\begin{abstract}
We have developed a radiant technique for substrate heating which we found particularly useful for the in situ deposition of high temperature superconducting films. Using this technique, large area $\mathrm{YBa}_{2} \mathrm{Cu}_{3} \mathrm{O}_{x}$ films were deposited, using off-axis sputter deposition, onto lanthanum aluminate and sapphire substrates, the latter using an yttria-stabilized zirconia buffer layer. For both types of substrates we were able to obtain c-axis oriented films. The dc and microwave properties of the films deposited on lanthanum aluminate are presented along with the dc measurements of the films deposited on sapphire. The motivation for employing this method of heating are discussed, along with our current plans for scaling up the process to produce even larger area films.
\end{abstract}

\section{INTRODUCTION}

The utilization of high temperature superconductors in advanced microwave devices requires techniques which yield reproducible deposition of high quality films of large area (3 to 4 inch diameter) films on microwave compatible substrates. Off axis sputter deposition [1]-[3] has been shown to be an effective deposition technique, but in order to obtain uniform large area films substrate rotation will probably be required. The in situ deposition of high quality $\mathrm{YBa}_{2} \mathrm{Cu}_{3} \mathrm{O}_{\mathrm{x}}$ films requires a substrate temperature in excess of $700^{\circ} \mathrm{C}$ and an effective oxygen partial pressure well in excess of $40 \mathrm{~m}$ Torr. This puts a number of requirements upon the substrate heater; it must uniformly heat a large area, be oxygen compatible and permit substrate rotation. Our current design does not yet permit substrate rotation and therefore limits us to 2 " diameter substrates.

Sapphire is a more attractive candidate microwave compatible substrate material than $\mathrm{LaAlO}_{3}$ due to its superior dielectric properties, greater strength (permitting a thinner substrate) and greater availability, especially for larger sizes. Because sapphire reacts with the superconducting oxides, it is necessary to first deposit a buffer layer which can be epitaxially grown on the sapphire, and which also has a lattice match to the superconducting film. For that purpose we are investigating yttria-stabilized zirconia as a buffer layer.

Manuscript received October 17, 1994.

\section{SuBSTRATE HEATING}

To in situ deposit c-axis oriented $\mathrm{YBa}_{2} \mathrm{Cu}_{3} \mathrm{O}_{\mathrm{x}}$ films it is necessary that the substrate be heated to above $700^{\circ} \mathrm{C}$ during the deposition process. This can be a rather challenging requirement in conjunction with the need for 2 " and larger diameter substrates. For small substrates (on the order of a centimeter) one can fairly easily attach a substrate to a heater block using silver paste and thus obtain the desired temperature. It is not difficult to obtain heating blocks that are oxygen compatible and can achieve temperatures of $800^{\circ} \mathrm{C}$ or more. It is more difficult to attach a $2 "$ diameter substrate to a heater block, with uniform thermal contact, and subsequently remove it after deposition. This is especially difficult when one is using substrates that are less than $250 \mu \mathrm{m}$ thick, a thickness that is required for higher frequency devices.

In order to avoid the problems associated with the bonding of a substrate to a heater, we pursued a radiant heating approach. By placing an opaque substrate in close proximity to a larger area heating block (Fig. 1.) one should be able to obtain good temperature uniformity. In order to radiantly heat an opaque substrate in vacuum to $740^{\circ} \mathrm{C}$, the heater block must be at about $930^{\circ} \mathrm{C}$, based on a very simple black body calculation. If a substrate is optically transparent then the substrate temperature will be significantly reduced. Therefore, practical radiant heating requires that the substrate be opaque, and preferably black. The method we used is described in the next section.

The introduction of a sputter gas mixture (100 to 200 mTorr) into the deposition chamber has two competing effects: it conducts heat from the heater to the substrate (increasing the substrate temperature) and it conducts heat away from the substrate (decreasing the substrate temperature). In order to achieve the required temperature control, it is necessary to determine the substrate temperature as a function of gas pressure. To do this a thermocouple was attached to the deposition side of an opaque substrate and the substrate temperature was measured as a function of both the heater block (control) temperature and the gas pressure. The results are plotted in Figure 2. We were surprised to discover that the substrate temperature was not a function of the gas pressure or oxygen/argon ratio, in the range of interest. It should be noted that this calibration does not include the heat generated by the $r f$ plasma. At these temperatures this should account for a relatively small and consistent effect, of the order of $10^{\circ} \mathrm{C}$. 


\section{HEATER BLOCK}

\section{2" SUBSTRATE} AND HOLDER
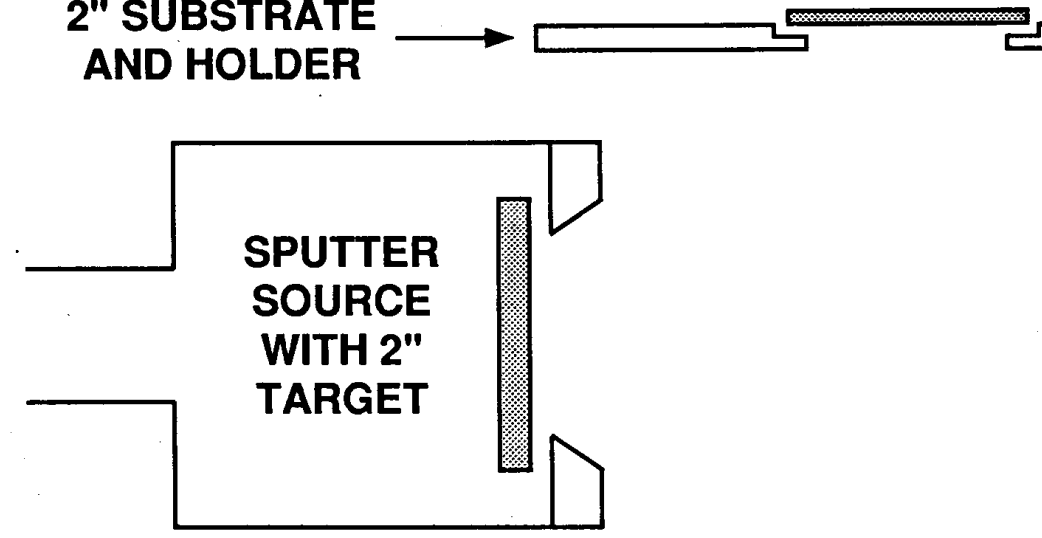

Fig. 1. Our off-axis sputter deposition geometry. A substrate (made opaque by a thick black film on its back side) is radiantly heated from the back, while the $\mathrm{YBa}_{2} \mathrm{Cu}_{3} \mathrm{O}_{\mathbf{x}}$ film is in situ deposited on the other side. The heater-substrate separation is approximately $1 \mathrm{~cm}$.

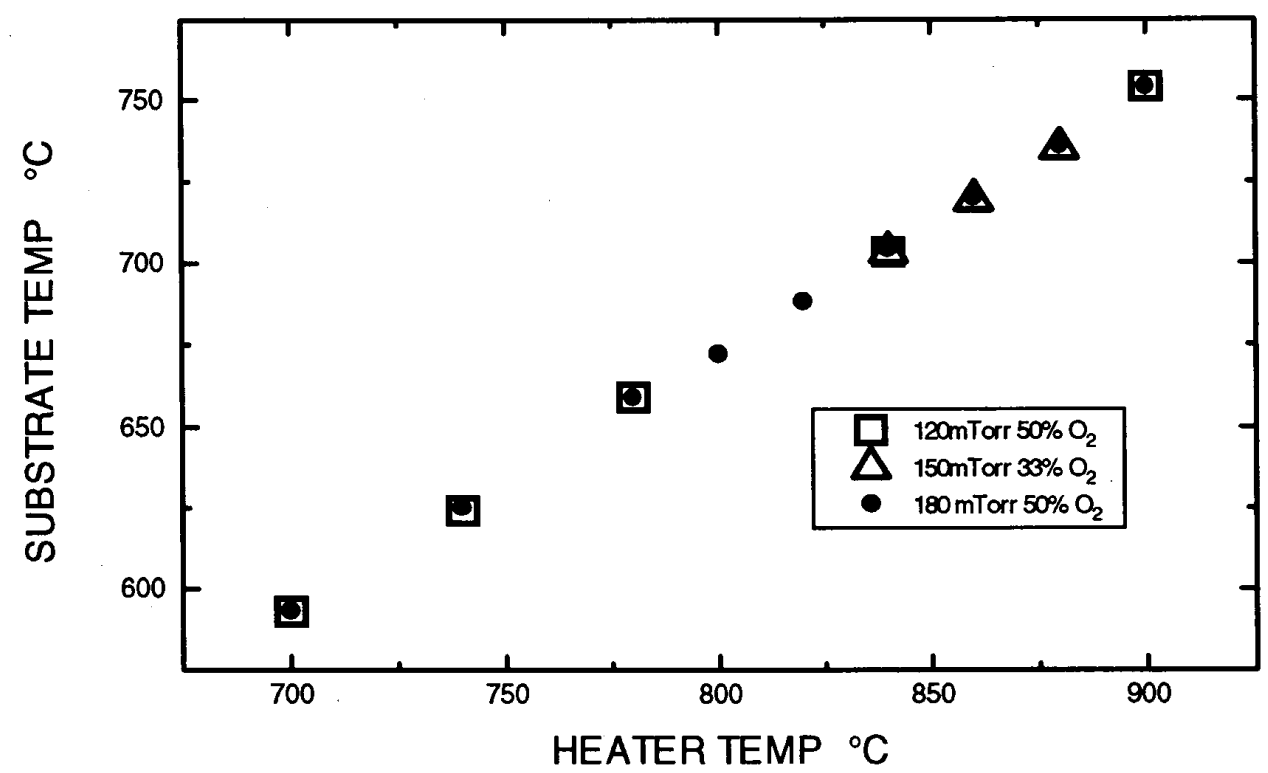

Fig. 2. Calibration of the substrate temperature as a function of the heater temperature for different gas pressures and mixtures. 


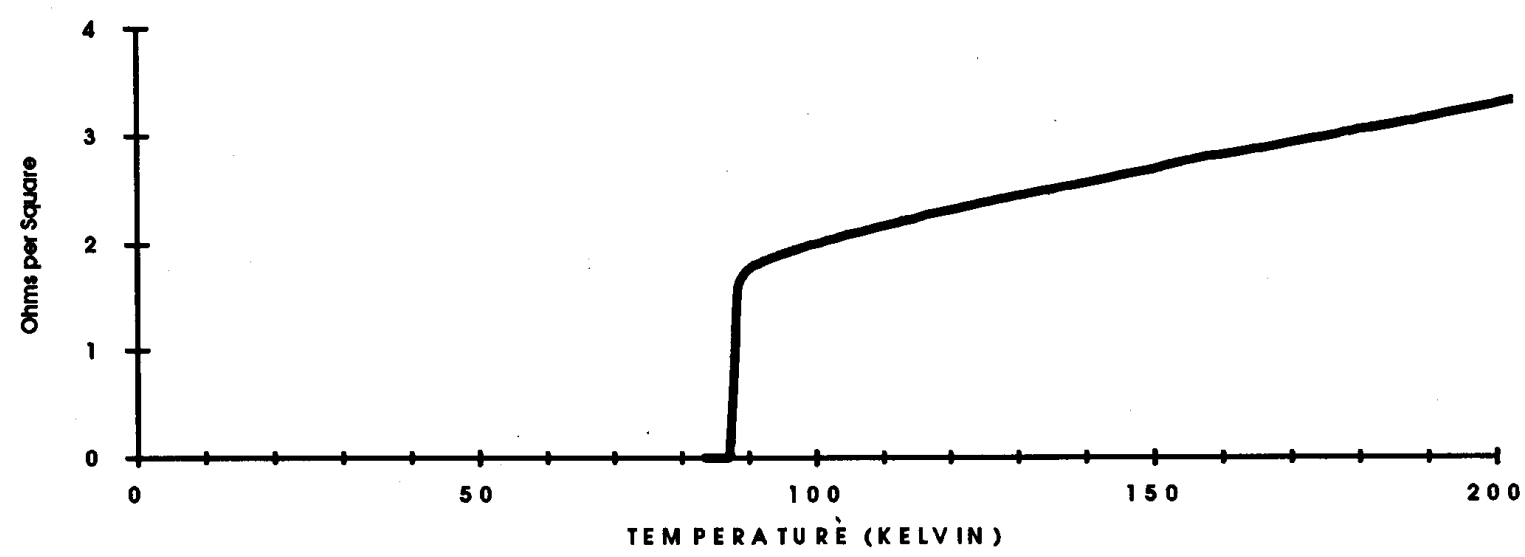

Fig. 3. Resistive transition of a $0.4 \mu \mathrm{m}$, c-axis oriented, $\mathrm{YBa}_{2} \mathrm{Cu}_{3} \mathrm{O}_{\mathbf{x}}$ film on a $\mathrm{LaAlO}_{3}$ substrate. The zero resistance temperature is approximately $87 \mathrm{~K}$.

\section{SUBSTRATE BLACKENING}

The $\mathrm{LaAlO}_{3}$ or sapphire substrates were made opaque by depositing 3 to $5 \mu \mathrm{m}$ of off-stoichiometry $\mathrm{Y}-\mathrm{Ba}-\mathrm{Cu}-\mathrm{O}$ or $\mathrm{CuO}$ on the back side (the side that will face the heater) of the substrate. This deposition was done in a diode sputter system using a 5" diameter ceramic target and a target to substrate distance of 3 to $5 \mathrm{~cm}$. The substrate was not heated during deposition, but it was subsequently annealed at $860^{\circ} \mathrm{C}$ in oxygen. The resulting material was black and opaque. Our requirement for the thickness was that no observable light could be seen through the film when viewed in an optical transmission microscope. We found that this blackening material can be readily removed chemically and replaced by a metallic ground plane.

\section{EPITAXIAL YBCO ON $\mathrm{LaAlO}_{3}$}

High quality $\mathrm{YBa}_{2} \mathrm{Cu}_{3} \mathrm{O}_{\mathrm{x}}$ films (of approximately $0.4 \mathrm{~nm}$ thickness) were in situ deposited on $\mathrm{LaAlO}_{3}$ substrates. A sputter gas pressure of approximately $140 \mathrm{mTorr}$ (typically comprised of $57 \%$ argon and $43 \%$ oxygen) was used. The $2 "$ if magnetron sputter source was run at 70 to 100 Watts. A heater block temperature of 880 to $900^{\circ} \mathrm{C}$ was used. After the deposition, the films were slowly cooled in $0.8 \mathrm{~atm}$ of pure oxygen.

The films were examined by electron microscopy and $x$ ray diffraction. The films are smooth, with features of less than 1 micrometer in size. $\mathrm{X}$-ray diffraction analysis indicates that they are c-axis oriented [4].

Electrical characterization of these films involved three measurements: surface resistance at $9.3 \mathrm{GHz}$ (using a sapphire resonator [5]), a dc measurement of the transition temperature and a dc measurement of the critical current density. The dc measurements typically give a zero resistance temperature, $\mathrm{T}_{\mathrm{c}}$, of 86 to $88 \mathrm{Kelvin}$ (Fig. 3.). Using a 20 micrometer wide bridge, patterned by wet chemical etching, a critical current density of approximately $1.2 \times 10^{6} \mathrm{~A} \mathrm{~cm}^{2}$ was measured at $77 \mathrm{Kelvin}$. Figure 4 . shows the inverse of the quality factor $(1 / Q)$ of the sapphire resonator as a function of temperature for a $\mathrm{YBa}_{2} \mathrm{Cu}_{3} \mathrm{O}_{\mathrm{x}}$ film on a $\mathrm{LaAlO}_{3}$ substrate, and for a thick copper film on silicon. As the surface resistance, $R_{S}$, is proportional to $1 / Q$, one sees that the $R_{S}$ of the superconducting film is less than copper by a factor of 30 or more at operating temperatures. Assuming a frequency squared dependence of $R_{S}$ for the YBCO film, and a linear dependence for the copper film, then at $20 \mathrm{GHz}$ the YBCO still has an $\mathrm{R}_{\mathrm{s}}$ that is over an order of magnitude lower than that of copper.

\section{SUPERCONDUCTOR ON SAPPHIRE}

For frequencies above $40 \mathrm{GHz}, \mathrm{LaAlO}_{3}$ is a less suitable substrate material. Due to its dielectric constant of around 25 , such frequencies require a substrate thickness which are not practical, less than $200 \mu \mathrm{m}$ at $40 \mathrm{GHz}$. Sapphire, on the other hand, has both a lower dielectric constant (around 10) and is less fragile than $\mathrm{LaAlO}_{3}$. In addition, sapphire is more economical than $\mathrm{LaAlO}_{3}$. The difficulty with the use of a sapphire substrate is that it reacts with the superconducting film. For this reason we began an investigation into the use of yttria stabilized zirconia (YSZ) as a buffer layer between the sapphire and the $\mathrm{YBa}_{2} \mathrm{Cu}_{3} \mathrm{O}_{\mathrm{x}}$ film.

The YSZ was deposited on r-plane sapphire using an onaxis diode sputter deposition process [6]. The sapphire substrate was first blackened, as previously described. A substrate temperature of over $650^{\circ} \mathrm{C}$ was required to obtain 


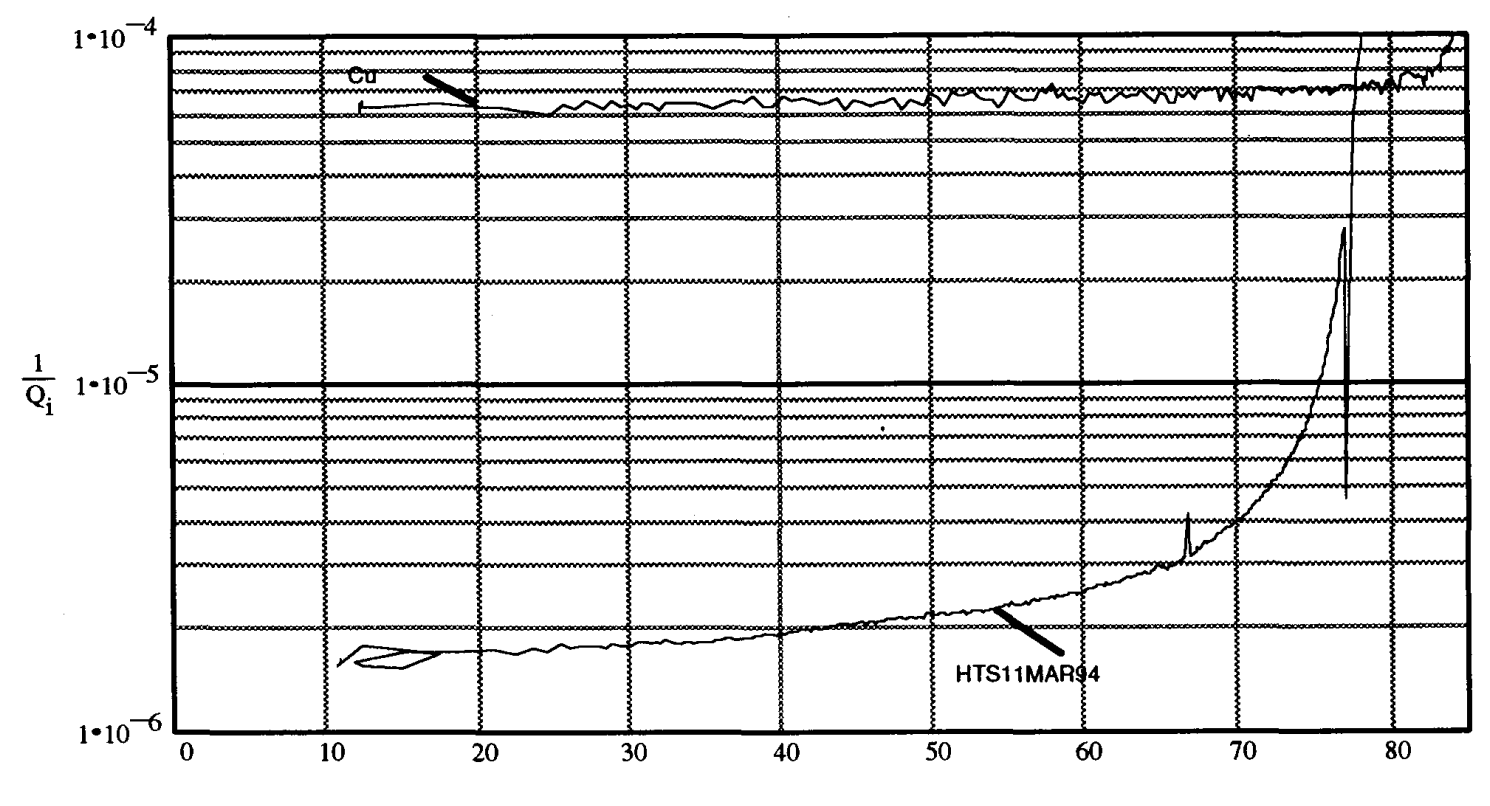

TEMPERATURE (KELVIN)

Fig. 4. Comparison of the surface resistance of $\mathrm{YBa}_{2} \mathrm{Cu}_{3} \mathrm{O}_{\mathrm{x}}$ film (HTS11MAR94) on $\mathrm{LaAlO}_{3}$ to that of a copper film, as a function of temperature. The measurement used a superconducting film (at lower temperature) on one end of a sapphire resonator with the sample on the other. Thus the copper data is only valid below about $80 \mathrm{~K}$. The actual value measured was the quality factor, $\mathrm{Q}$, of the resonator, which is inversely proportional to the surface resistance of the two end walls. Thus the superconducting film data represents an upper limit to its actual value.

(100) oriented YSZ on r-plane sapphire [6]. After depositing the YSZ buffer layer, the sapphire substrate was put into the off-axis deposition system and the superconducting film was deposited using the same conditions used for YBCO deposition on a $\mathrm{LaAlO}_{3}$ substrates (as described in the prior section).

These films were characterized by $\mathrm{x}$-ray diffraction and $\mathrm{dc}$ measurements. X-ray diffraction confirmed that the YSZ was in a (100) orientation on the r-plane sapphire and that the subsequent $\mathrm{YBa}_{2} \mathrm{Cu}_{3} \mathrm{O}_{\mathrm{x}}$ film was $\mathrm{c}$-axis oriented. The $\mathrm{T}_{\mathrm{c}}$ of these films was 2 to $3 \mathrm{~K}$ lower and the critical current density, at $77 \mathrm{~K}$, was about one half that of films deposited on $\mathrm{LaAlO}_{3}$. These results are recent and it is likely that the quality of the superconducting films on YSZ is not optimized.

\section{CONCLUSIONS}

A technique has been shown for the uniform heating of large area substrates for in situ deposition of $\mathrm{YBa}_{2} \mathrm{Cu}_{3} \mathrm{O}_{\mathrm{X}}$ or other similar materials. Substrate heating to over $750^{\circ} \mathrm{C}$ is achieved by locating a large area heater block in close proximity to a smaller area substrate. In order to efficiently absorb the radiant heat, the substrate is made black and opaque by the deposition of a thick YBCO or CuO film on its back side. Our current sputter deposition system is limited to 2 " diameter substrates because the substrate holder cannot rotate. A new system, about to be installed, has substrate rotation and larger ( $3 ")$ sources. With these improvements, we expect to be able to uniformly heat and deposit $\mathrm{YBa}_{2} \mathrm{Cu}_{3} \mathrm{O}_{\mathrm{x}}$ on substrates of over 3" diameter. We continue to work on improving the $\mathrm{YBa}_{2} \mathrm{Cu}_{3} \mathrm{O}_{\mathrm{x}}$ films on sapphire using YSZ and other buffer layer materials.

\section{REFFERENCES}

[1] R. L. Sandstrom, et al., Reliable single-target sputter process for high-temperature superconducting films and devices, Appl. Phys.Lett. vol. 53, pp. 444-446, 1988.

[2] C. B. Eom, et al., In situ grown $\mathrm{YBa}_{2} \mathrm{Cu}_{3} \mathrm{O}_{7-\mathrm{d}}$ thin films from singletarget magnetron sputtering, Appl. Phys. Lett, vol. 53, pp. 595-597, 1989.

[3] N. Newman, B. F. Cole, S. M. Garrison, and K. Char, Double gun offaxis sputtering if large area $\mathrm{YBa}_{2} \mathrm{Cu}_{3} \mathrm{O}_{7-\mathrm{d}}$ superconducting films for microwave applications, Appl. Phys. Lett., vol. 57, pp. 520-523, 1990.

[4] A. J. Drehman, et al. Large area high temperature superconducting films for microwave applications, IEEE Mohawk Valley Section Proceedings on Dual Use Technologies and Applications, part I, pp. 333-338, 1994

[5] Zhi-Yuan, et al., High $T_{c}$ superconductor-sapphire microwave resonato with extremely high Q-values up to $90 \mathrm{~K}$, IEEE Trans. Microwave Theory and Techniques, vol. 40, pp. 2424-2431, 1992.

[6] J. H. Kroese, A. J. Drehman and J. A. Horrigan, Reduced YSZ deposition temperatures for $\mathrm{YBa}_{2} \mathrm{Cu}_{3} \mathrm{O}_{7} / \mathrm{YSZ}$ thin films on sapphire, unpublished. 